\title{
Improving Usability by Adding Security to Video Conferencing Systems
}

\author{
April Slayden Mitchell, Alan H. Karp \\ Hewlett-Packard Laboratories. 1501 Page Mill Rd, Palo Alto, California, USA \\ \{april.mitchell, alan.karp\}@hp.com
}

\begin{abstract}
Many video conferencing solutions exist in the market today and many new ones are being introduced. In striving to provide an experience to users as close to "being there" as possible, two major design issues must be considered: security and ease of use. In this paper, we describe a method for designing a "One Space" video conferencing user interface that reveals security information to the users while reducing the complexity of the user experience.
\end{abstract}

Keywords: Usability, Security, User Interface Design

\section{Introduction}

Many desktop video conferencing systems are being sold as a way to reduce the need for business trips. Shortcomings of these systems are apparent to many consumers; we'll focus on two of them here. The first drawback is a confusing interface that makes many conferencing applications too complicated to use. The second is that private discussions cannot be shared via the public internet for fear of leaks and vulnerability. While encryption protects the data being transmitted, users are also concerned about off-camera "watchers" and over the phone "listeners". In this paper, we describe a user interface that addresses both of these issues.

\section{Motivation}

In comprehensive video conferencing solutions, such as HP's Halo ${ }^{1}$ and Cisco's TelePresence ${ }^{2}$, it is important that users are able to operate comfortably while in the room and are able to make the same assumptions about security as they are in a standard conference room.

At the center of the complexity and confusion for users of standard video conferencing applications and conferencing equipment is the user interface. Too often the interface consists of menus within menus. While this pattern is familiar from

\footnotetext{
${ }^{1}$ HP Halo: http://www.hp.com/halo/index.html

${ }^{2}$ Cisco Telepresence:

http://www.cisco.com/en/US/netsol/ns669/networking_solutions_solution_segment_home.html
} 
desktop computing, it is nevertheless confusing for occasional users, leading them to ask questions such as: "Which menu do I look in to find the phone?" and "Where's the option for sharing my display?”

Nested menus are also detrimental to security. Without extensive customization, users are often presented with many options that the security policy prevents them from using, thus revealing protected information and introducing confusion (see principle of expected ability in [2]). In order for a video conferencing application to be a success, the user interface must be understandable by a novice, warrant repetitive use, and reveal the aspects of security relevant to the participants. User studies showed that knowing who was listening was the top concern. Clearly, the introduction of cameras and microphones into a space invalidates the "assumption" of private communication. We took privacy strongly into consideration in developing our model for a secure video conferencing solution interface. The theme of our solution can be summarized by the term "Revelation", revealing the presence of "watchers" and "listeners" as well as making any action taken visible to all participants.

\section{The "One Space” Metaphor}

A video conferencing system consists of one or more physical locations (rooms containing the video conferencing equipment) connected over a network. In a "One Space” video conferencing system, both the interface and room design encourage people to act as if the physical room is a single location in which all participants are present. All rooms consist of the same equipment and physical layout, including a center table, chairs, lighting, and color scheme. The rooms are not customized based on company or location, thus adding to the illusion of a shared environment.

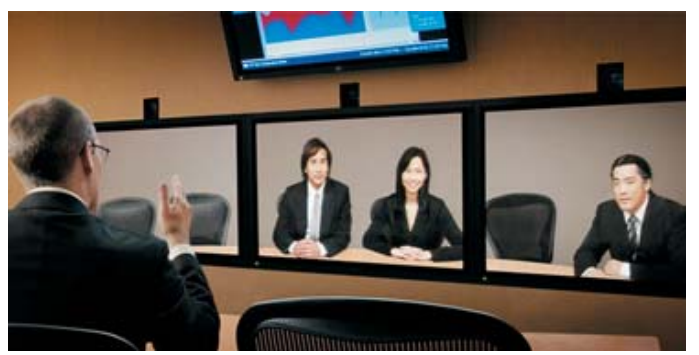

Fig. 1. This image shows the physical layout of an HP Halo video conferencing room.

In addition to various microphones and speakers, each room contains video display screens for showing other attendees and an additional display for showing the shared interface (see Figure 1 for an example from HP Halo). This display contains means for controlling all of the physical devices available in each room, including the cameras, PCs, etc. This shared display is considered an extension of the table desktop and is visible to all attendees in every room.

In the One Space metaphor, it is very important that all rooms always view the same user interface. Attendees in any room can control the interface by using a device 
such as a mouse, which allows full control of the interface and its actions are visible to everyone, regardless of room. All participants, including those dialing in over the phone, and all resources, such as overhead cameras, are represented by icons in a virtual room. By presenting a schematic representation of the rooms and functions involved in the meeting, we enforce the One Space metaphor.

Encryption and the use of private networks can address many security concerns. They can not answer questions such as: "Who is on the phone?", "Is there a helpassistant listening?”, "Is the door to the room open?”, and "Are there people in the room who are off-camera?” Our One Space user interface addresses these questions. There is no way through the interface to connect to and "listen in" to a conference uninvited or unannounced. All video links must be explicitly accepted or declined. The audio doesn't start until the video is displayed. All attendees who dial in to a conference line are represented by a uniquely identified icon. Similarly, the audio connection with the help-assistant is always represented on the user interface by a representation of an occupied or unoccupied desk.

\section{Implementation}

Our proposed One Space interface, shown in Figure 2, appears on the shared display in each room. ${ }^{3}$ It consists of a schematic representation of the physical space and shows virtual devices, such as help information, which are available. Dial-in participants can access this view via a web browser. The numbered callouts in the figure do not appear on the actual interface; they appear only to facilitate the description.

The spatial view of the One Space interface, in which all equipment, devices, etc. are represented as icons, removes the need for traditional nested menus. All icons are pictorial and represent common objects such as a table, a phone, or a camera. Mouse tips can provide alternative denotations. The shared control of the space is an essential element in making the interface more secure and easier to use because there are no hidden actions by any connected parties, and there is no need to worry about the learning curve due to using a room in a different location.

\subsection{Revelation Icons}

All video conference rooms connected will be represented on the One Space interface. Figure 2 represents an event in which two rooms are connected; however, the table as indicated by callout 1 can be expanded to show an event with more rooms or reduced to show only one side of the table when the room is being used locally. As shown in callout 6 , the location and company information for each room is displayed on the interface. The public information appearing on the interface can be configured during booking: such as, the attendee list, the conference telephone number, and each room's

\footnotetext{
${ }^{3}$ The ideas contain herein are not details of any video conferencing product by HP or any other company. They are proposals for designing a more usable, more secure video conferencing interface.
} 
LAN access (internal or external). If there are network and/or phone connections in a room, then their existence and activity are viewable via the interface. In Figure 2, a laptop is shown on the table (callout 10), which denotes that it has been connected to the network within Company B's room.

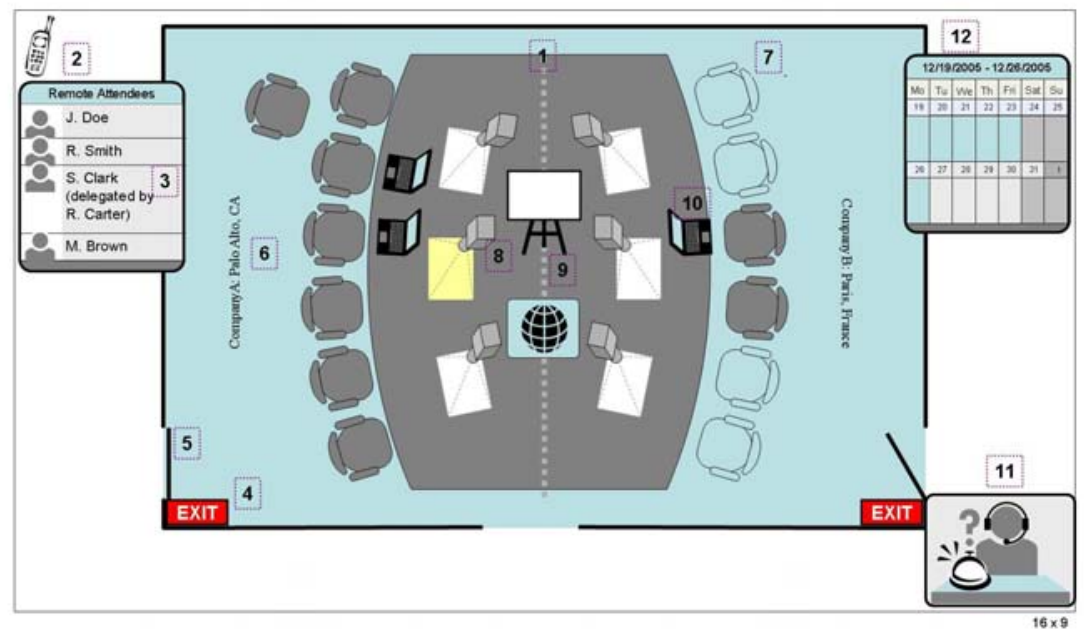

Fig. 2. This is an example of a One Space user interface for a video conference connection between two physical locations with remote participants dialed in via a conference line.

In callout 2, remote attendees who are dialed in over the phone are recognized on the interface. This information is tracked through a per-meeting/per-person pass code which each invited member receives. Callers can enter their names via keypad or using speech recognition software. Each name is compared with the name of the responsible party. If the name is different from the name associated with the PIN used, the system recognizes that this person is a delegate of the responsible party and lists the joining party's name as well as the responsible party's name (callout 3).

Every physical seat in each room is shown on the interface as a chair icon colored based on whether or not the seat is currently occupied. Additionally, on-camera seats are distinguished from off-camera seats. Callout 7 shows an off-camera, unoccupied seat. This detection can be done by weight sensors placed on the chair to determine occupancy as well as by camera tracking tools which could identify chairs brought into the room or extra people standing off-camera [1].

In addition to seats in the room, an icon representing the door to the room (callout 5) will also appear on the interface. This icon changes to indicate whether or not the actual physical door is open or closed in order to reveal if persons located outside of the room can easily hear or see participants in the video conference.

\subsection{Interaction Icons}

In addition to icons on the interface which reveal attendance and connection information, there are other icons which allow users in either room to control 
conference actions. For example, callout 4 indicates an Exit sign which is how a room leaves the conference. All other rooms will still stay connected and the table on the One Space interface will adjust to reflect that one room has left the event.

Several icons representing overhead cameras are shown on the interface. The interface also reveals the area on the table that will be illuminated if it a particular camera is activated. Once a camera or device is activated, the area on the One Space interface table (callout 8) will be shaded a different color to reflect the portion of the physical table that is on camera.

Icons that appear in the center of the table (callout 9) represent virtual devices which can be accessed. These may include a shared web browser or whiteboard. The icon denoted by callout 11 represents the help desk. It should only show the desk as occupied when the help help-assistant is online and can interact with the meeting attendees. When the help-assistant is not connected, the icon should appear as an unoccupied desk with a bell. Any user may click on the bell to contact the helpassistant.

A calendar, as indicated by callout 12 , should be accessible when in a video conference. This calendar shows the availability of all rooms currently connected. Through this interface, attendees are able to schedule a follow-up event with the same rooms and the same attendees, but not access any other room's calendars or change the attendee list.

\section{Conclusion}

Utilizing the One Space metaphor for the user interface both enhances meeting attendees' feelings of occupying a shared physical space and provides confidentiality assurances, such as knowing at all times who else is present in the connected rooms. The schematic of the One Space interface makes using the various devices in connected rooms intuitive while enhancing security by revealing physically absent listeners at all times. Similarly, the pictorial representation of all devices, attendees, etc. and the consistency of the interface regardless of room locale makes repetitive use simple. HP has utilized portions of the One Space interface guidelines in designing the interface for Halo, and internal studies have shown successful use by novices and overall improved user experience. Utilizing the One Space metaphor when designing video conferencing interfaces will provide more visible security assurances and support repetitive use, all while enabling even a novice to use the system to its fullest extent.

\section{References}

1. Harville, Michael; Li, Dalong.: Fast, Integrated Tracking and Activity Recognition with Plan-View Templates from a Single Stereo Camera. Proceedings of the IEEE Computer Vision and Pattern Recognition Conference. Washington, DC (2004).

2. Yee, K.: User Interaction Design for Secure Systems. Proceedings of the Fourth International Conference on Information and Communications Security. Singapore (2002). 